\title{
Role and management of extracorporeal life support after surgery of chronic thromboembolic pulmonary hypertension
}

\author{
Sofia Martin-Suarez ${ }^{1}$, Gregorio Gliozzi ${ }^{1}$, Mariafrancesca Fiorentino ${ }^{1}$, Antonio Loforte ${ }^{1}$, Valentina Ghigi ${ }^{2}$, \\ Marcello Di Camillo ${ }^{3}$, Nazareno Galiè ${ }^{4}$, Davide Pacini ${ }^{1}$ \\ ${ }^{1}$ Cardiac Surgery Department, ${ }^{2}$ Cardiac Surgery Anesthesia Department, ${ }^{3}$ Clinical Perfusionist, ${ }^{4}$ Cardiology Department, Pulmonary Hypertension \\ Unit, Bologna University, St. Orsola Hospital, Bologna, Italy \\ Correspondence to: Sofia Martin-Suarez. Cardiac Surgery Department, Bologna University, St. Orsola Hospital, Padiglione CTV num 23, Via \\ Massarenti, 9 CAP 40138, Bologna, Italy. Email: docsofi74@hotmail.com.
}

\begin{abstract}
Background: Pulmonary endarterectomy (PEA) is a surgical intervention reserved for patients with chronic thromboembolic pulmonary hypertension (CTEPH). In some cases, temporary circulatory support [extracorporeal life support (ECLS)] is required after PEA. Rates of ECLS requirement varies between centers. Reasons for institution of ECLS include respiratory failure, cardiac failure (or both respiratory and cardiac failure), bleeding, and reperfusion edema. This article reviews the experience of ECLS after PEA from the current literature, as well as our own institution's experience as a CTEPH multidisciplinary center.

Methods: A literature review was conducted along with a retrospective chart review from 15 years of our PEA program.

Results: The literature demonstrates many different approaches are used for mechanically supporting patients who develop complications after PEA. Variations in approach stem from differing indications such as, respiratory failure rather than hemodynamic compromise (or vice versa), time of implantation (immediately in operating room or delayed after surgery) and many other causes. In our center, $12.3 \%$ (19/154) of patients need ECLS with extracorporeal membrane oxygenator (ECMO) after PEA procedure. Implantation was mainly in the operating room before or immediately after weaning from cardiopulmonary bypass and mostly peripheral cannulation was used. ECMO lasted an average of $11 \pm 8$ days. And 52.6\% (10 of 19 patients) of patients were weaned from ECLS and of this, 70\% (7 of 10 patients) were discharged.

Conclusions: In some cases of PEA, ECLS is needed post-operatively. Expert teams should consider this possibility pre-operatively based on predisposing characteristics. The need for ECMO shouldn't be "di per se" a contraindication to surgery but might be considered in the surgical risk estimation. The ideal setup is not fixed and depends on the center's practices as well as indication. Even though complications do occur with ECMO, in general, results are good, being a bridge to further recovery of pulmonary hypertension $(\mathrm{PH})$ or also to transplantation.
\end{abstract}

Keywords: Extracorporeal membrane oxygenator (ECMO); pulmonary endarterectomy (PEA); deep hypothermic arrest; pulmonary hypertension $(\mathrm{PH})$

Submitted Oct 02, 2018. Accepted for publication Dec 03, 2018.

doi: 10.21037/acs.2019.01.02

View this article at: http://dx.doi.org/10.21037/acs.2019.01.02

\section{Introduction}

Chronic thromboembolic pulmonary hypertension (CTEPH) is an uncommon disease, infrequently seen after acute pulmonary embolism (APE) and associated with severe morbidity and mortality (1). CTEPH represents nearly $19 \%$ of patients referred to dedicated centers with PH (2). The pathology of CTEPH is characterized by fibrotic progression of acute fresh clots, leading to vessel walls becoming fibrotic and narrowed, with luminal 
occlusion. Concomitantly, in the non-occluded territory, vessels regulate excessive flow by vasoconstriction, leading to histological changes, which contribute to $\mathrm{PH}$ and may not be immediately reversible and in some cases, permanent even after reopening of occluded arteries.

CTEPH is the only variant of pulmonary hypertension $(\mathrm{PH})$ with a surgical and curative treatment-pulmonary endarterectomy (PEA). Results are satisfying in terms of low postoperative morbidity and mortality as well hemodynamic recovery (3). Patient selection for treatment of CTEPH with PEA should always be discussed in a multidisciplinary team meeting.

Despite careful patient selection for PEA and the sophisticated and systematic techniques used in dedicated centers, some patients may suffer from life-threatening postoperative complications requiring extracorporeal life support (ECLS).

One of the most common complications after PEA is reperfusion edema of the lung parenchyma which occurs in between $5-20 \%$ of patients (4). The mechanism of lung parenchyma reperfusion edema is not clear but it is believed that there is a capillary-alveolar membrane dysfunction in those territories of reperfused lung parenchyma previously occluded. Variable degrees of reperfusion edema are frequently observed after PEA. In uncomplicated situations, supportive management with mechanical ventilation and diuretics is adequate. However, in complicated cases with persistent respiratory failure, ECLS may be necessary.

In some cases, patients suffer from residual $\mathrm{PH}$, which is typically limited to the early postoperative period and can be observed either in patients with good surgical results due to the reperfusion edema previously described or in patients with extensive peripheral and micro-vessel disease. In cases of micro-vessel disease, fibrotic thrombus is not surgically removable because it is located distally in small caliber vessels. These situations can be very difficult to identify before the operation (5) and together with surgical trauma and reperfusion edema, can lead to acute cardiorespiratory deterioration. In all these situations, different degrees of respiratory failure can additionally be accompanied by right ventricular failure.

Endobronchial/parenchymal bleeding is the most critical (and often, fatal) serious complication (6). During the procedure, fragility or perforation of the pulmonary vessels can be detected using the "bubble" technique. If bubbles are identified during soft ventilation in distal pulmonary branches, bleeding will probably occur and the branch should be occluded with application of surgical sealant.
In all the previous situations (which of course, can coexist), medical management could be insufficient, and temporary ECLS should be considered.

As reported from a group in San Diego (4), respiratory and oxygenation capabilities are severely compromised in some cases but cardiac function remains normal, hence, veno-venous cannulation is preferred. Also known as extracorporeal carbon dioxide removal $\left(\mathrm{ECCO}_{2} \mathrm{R}\right)$, it has many advantages, including minimally invasive peripheral cannulation, preservation of pulsatility, preload and stroke volume. Successful weaning off ECLS was achieved in $40 \%$ of patients with a survival of $30 \%$ (4).

Right ventricular failure is a complication that can occur in isolation or with other complications since pulmonary vascular resistance (PVR), cardiac output (CO) and gas exchange are intimately interrelated. The PEA group at Cambridge considers that in order to interrupt this vicious circle, veno-arterial extracorporeal membrane oxygenation (ECMO) is indicated. With veno-arterial configuration, PVR decreases due to better oxygenation and reduced hypoxic vasoconstriction. Right ventricular pre- and afterload is reduced, allowing better recovery (7). Moreover, blood flow through the pulmonary vessels is minimized while maintaining pulsatility. In the Cambridge PEA group, the incidence of post-PEA ECMO was 5.5\% (7/127 patients) with $71.4 \%$ of patients $(5 / 7)$ weaned and $57.1 \%$ (4/7 patients) discharged. While they cannulate the right atrium and aorta (central cannulation), other groups favor the peripheral approach $(8,9)$. The Vienna group (9) reports a rate of ECMO weaning of $90.3 \%$ (28 of 31 patients on ECMO), while the overall need for ECMO was $19.3 \%$ (31 patients of 161 PEA population). Of these 31 patients, 2 were on ECLS pre-operatively. The survival rate of patients requiring ECMO was $67.7 \%$. Patients unable to be weaned were listed and transplanted. This is reflected in France, where policy includes consideration of ECLS as a bridge to transplantation (BTT) (3). The authors reported a rate of ECLS after PEA of 3.7\% (31/829), including 23 with ECMO as a BTR (bridge to recovery) and 8 as a BTT. Survival of both groups (BTT and BTR) was $50.0 \%$ and $47.8 \%$ respectively.

In general, central cannulation might be indicated when institution of ECLS is necessary in the operating theater after an unsuccessful weaning from cardiopulmonary bypass. When the clinical and hemodynamic condition deteriorates in the hours after the surgery (when the patient is already in the intensive care unit) it appears reasonable to implant a peripheral veno-venous ECMO when oxygenation is 
the only complication or veno-arterial ECMO when hemodynamic compromise is the prevailing issue.

In those unusual cases of massive bronchial/pulmonary bleeding after PEA (0.5-2\%) (10), management can be arduous- in these scenarios, ECMO is a well-established life-saving tool. The benefit of ECMO rather than CPB is that circuits are heparin-coated and the patient's coagulation profile can be completely restored, with minimal risk of clot formation inside the oxygenator while allowing for resolution of hemorrhage $(11,12)$.

In the following sections we report our own experience and policy in patients treated with ECLS after PEA.

\section{Methods}

From December 2003 to July 2018, 154 PEA procedures have been carried out at our institution. All patients selected for PEA were discussed in a multidisciplinary team including heart surgeon, cardiologists, and radiologist. Patients are referred to our specialized $\mathrm{PH}$ center, often with the imprecise diagnosis of $\mathrm{PH}$ without underlying etiology. Diagnostic workup included ventilation/perfusion scan, echocardiogram, lung parenchyma angiographic evaluation with CT scan and right heart catheterization. When a definitive diagnosis of CTEPH was made, angiography was performed to evaluate operability. Six meter walking distance test (6MWD), spirometry with DLCO, and coagulation tests completed the diagnostic workup. Intervention of PEA was considered when thrombotic material was surgically accessible and the number of vessels significant (more than 50\%). The proportion of occlusion and degree of $\mathrm{PH}$ was taken into account, since irrelevant or very distal involvement with very high pulmonary pressure (disproportionate $\mathrm{PH}$ ) might indicate distal vessel diseasea negative prognostic factor which increases the risk of postoperative ECLS. In Figure 1, we report one example of a patient with high PVR (more than 14 Wood units) and a bad anatomy for PEA in terms of preoperative pulmonary angiography and surgical specimen. Definitive decisions are made in the context of the patient's risk profile: age, general conditions and associated pathologies all need to be considered. All patients received oral anticoagulation before the operation for at least three months and in a number of patients, oral pulmonary vasodilators [such as of endothelin receptor antagonists and/or a selective phosphodiesterase 5 inhibitor (sildenafil)] were also used.

At the beginning of our experience we adopted our own protocol of $\mathrm{CPB}$ management in order to avoid circulatory arrest and deep hypothermia. This was successfully performed in 49 cases (13). After this initial experience we decided to perform PEA with intermittent periods of deep hypothermic circulatory arrest [as guidelines recommend (14)].

ECMO indications were: (I) respiratory failure and hypercapnia when pulse oximetry was less than 90\%; (II) less than $20 \mathrm{mmHg}$ of difference between mean systemic and pulmonary pressure; (III) evident echocardiographic signs of severe right ventricular dysfunction and; (IV) biochemical and clinical negative data like lactic acidosis or oliguria. In some cases, inhaled nitric oxide (NO) was initiated when $\mathrm{PH}$ was unresolved. Norepinephrine was the only vasopressor used. Veno-arterial ECMO was used in all but one case given different degrees of both hemodynamic and respiratory failure occurred. ECLS was indicated in any case where bronchial bleeding occurred. We strongly believe that with the veno-arterial approach, the chance of right ventricular function recovery and improved reperfusion edema is higher since right ventricular after-load and pulmonary flow are more effectively regulated by the ECLS. Even if influential authors (15) have reported the benefit of pulmonary artery venting, we do not believe it is mandatory. Even by dramatically reducing the left ventricular filling, the interventricular septum shifting leftward would increase right ventricular ballooning and jeopardize its recovery. ECMO was implanted in the operating room when $\mathrm{CPB}$ was unable to be weaned. Peripheral cannulation (veno/arterial femoro-femoral cannulation) was our choice in 16 of 19 cases. In one case, arterial inflow was switched from the femoral artery to the right subclavian artery because of "harlequin syndrome" (16). In all cases, we used pre-coated cannulas and circuit lines. For arterial cannulation, surgical exposure of the femoral vessels was used along with the smallest cannula for the patient's BMI to avoid distal limb ischaemia. When signs of distal malperfusion were recognized, adjustments were made so adequate distal limb perfusion was achieved. The circuit comprised coated lines (Bioline Coating Maquet Getinge Group), a hollow fiber oxygenator of polymethylpentene (Quadrox Maquet Getinge Group) (Rotaflow Maquet Getinge group) and a centrifugal pump connected to heat exchanger. The ACT (Activated Clotting Time) target was around 180 to 200 seconds. Anticoagulation is not reversed when converting patients from $\mathrm{CPB}$ to ECMO. ECMO flow is maintained as low as possible in order to keep pulmonary pulsatility and reduce the risk of intravascular thrombosis. Also, mean pulmonary artery pressure (PAPm) was maintained as low as possible (nearly $20-30 \mathrm{mmHg}$ 

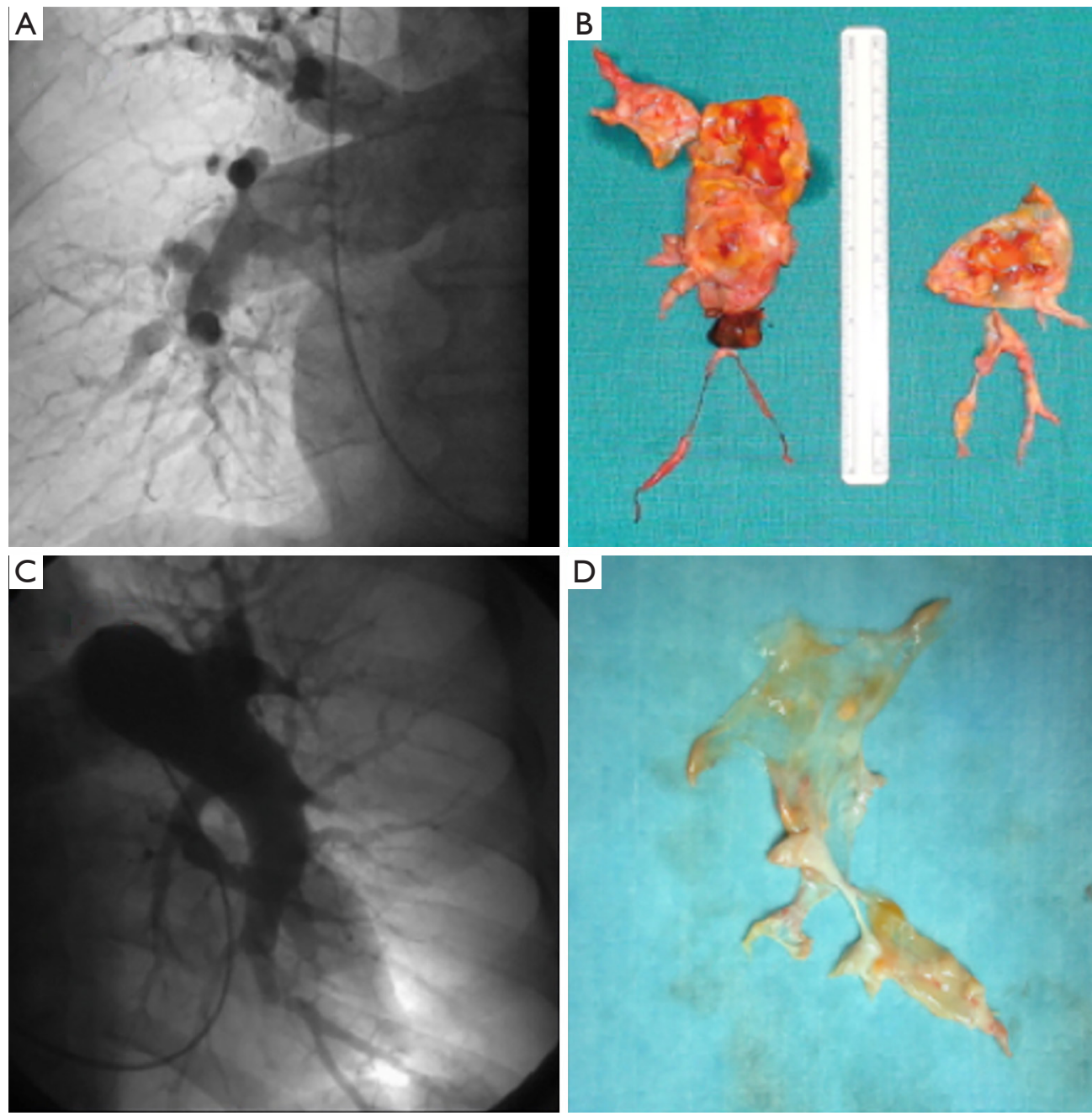

Figure 1 (A) Preoperative angiography of right pulmonary artery, anteroposterior projection. Proximal occlusion and vessel tortuosity is documented. (B) Surgical specimen confirming angiographic information. This would be considered a "good case for PEA". (C) Left pulmonary artery preoperative angiography, anteroposterior projection. There is a lack of distal and sub-pleural vessels. Those with a PVR more than $14 \mathrm{WU}$, might correlate with distal and micro vessel pathology, which is less reversible after PEA, increasing the risk of residual high PVR and need of ECLS. This suspicion is confirmed by surgical specimen (D). PEA, pulmonary endarterectomy; PVR, pulmonary vascular resistance; ECLS, extracorporeal life support.

less than the mean systemic arterial pressure (SAPm), i.e., PAPm less than 30 if SAPm of $60 \mathrm{mmHg}$ ). A lack of pulsatility is negatively correlated with RV function (17). This correlation has been reported in other fields but not in CTEPH, where the right ventricle is typically hypertrophic. Further evaluation and evidence of PAP pulsatility index utility might be analyzed in PEA patients.

Drains are left in place and the sternum is definitively closed. Frequent supervision of leg perfusion, diuresis preservation and surveillance for cardiac tamponade are mandatory. Patients should be maintained with a negative fluid balance and stable hemoglobin and coagulation parameters. Control of PAP can also be obtained with inhaled Nitric oxide and the administration of sildenafil. Mechanical ventilation parameters must include reduced tidal volume $(<5 \mathrm{~mL} / \mathrm{Kg})$, low peak airway pressure $(<25 \mathrm{mmHg})$ and variable degrees of positive end-expiratory pressure (PEEP). Inspired oxygen concentrations should be maintained lower than 0.7 in order to avoid oxygen toxicity. ECMO weaning should be performed as soon as possible depending on the patient's condition. Weaning should be progressive: increasing ventilatory support while 


\begin{tabular}{|c|c|c|c|}
\hline Variable & ECLS (n=19), N (\%) & No ECLS (n=125), N (\%) & $\mathrm{P}$ \\
\hline Age (years) & $53.42 \pm 15.75$ & $57.69 \pm 15.68$ & 0.27 \\
\hline BSA & $1.72 \pm 0.22$ & $1.8 \pm 0.23$ & 0.16 \\
\hline Male & $8(42.1)$ & $46(36.8)$ & 0.456 \\
\hline NYHA III-IV & $18(94.7)$ & $81(64.8)$ & 0.009 \\
\hline $\mathrm{O}_{2}$ therapy & $8(42.1)$ & 35 (28.0) & 0.169 \\
\hline Preoperative vasodilators & $12(63.2)$ & $80(64.0)$ & 0.037 \\
\hline Distal anatomy lesion & $4(21.1)$ & $6(4.8)$ & 0.021 \\
\hline Redo & $5(26.3)$ & $6(4.8)$ & 0.001 \\
\hline Emergency & $2(10.5)$ & $2(1.6)$ & 0.027 \\
\hline PAPs $(\mathrm{mmHg})$ & $93.84 \pm 19.58$ & $84.38 \pm 19.01$ & 0.046 \\
\hline PAPd $(\mathrm{mmHg})$ & $26.47 \pm 8.3$ & $26.89 \pm 12.11$ & 0.87 \\
\hline PAPm $(\mathrm{mmHg})$ & $50.63 \pm 13.12$ & $49.15 \pm 13.33$ & 0.65 \\
\hline PVR (UW) & $10.93 \pm 3.52$ & $8.84 \pm 3.71$ & 0.023 \\
\hline $\mathrm{Cl}\left(\mathrm{L} / \mathrm{min} / \mathrm{m}^{2}\right)$ & $2.41 \pm 0.46$ & $2.67 \pm 0.77$ & 0.166 \\
\hline $\mathrm{CO}(\mathrm{L} / \mathrm{min})$ & $4.02 \pm 0.87$ & $4.83 \pm 1.55$ & 0.031 \\
\hline CBP time (min) & $340.95 \pm 68.78$ & $279.29 \pm 70.95$ & 0.001 \\
\hline Cross clamp time (min) & $186.74 \pm 43.01$ & $163.73 \pm 49.97$ & 0.059 \\
\hline Circulatory arrest time (min) & $21.05 \pm 24.17$ & $17.52 \pm 18.31$ & 0.455 \\
\hline VR G1 (UW) & $6.51 \pm 5.48$ & $3.82 \pm 1.78$ & $<0.001$ \\
\hline
\end{tabular}

ECLS, extracorporeal life support; BSA, body surface area; PAP, systolic pulmonary artery pressure; PAPd, diastolic pulmonary artery pressure; PAPm, medium pulmonary artery pressure; PVR, pulmonary vascular resistance; $\mathrm{Cl}$, cardiac index; $\mathrm{CO}$, cardiac output; CBP, cardio-pulmonary bypass; G1, pod (post operative day) 1.

reducing ECMO flow and closely checking oxygenation, hemodynamic, laboratory and radiological parameters.

\section{Results}

ECLS was needed in $12.3 \%(19 / 154)$ patients. In those 44 cases performed with moderate hypothermia, ECMO was needed in 8 cases (18.2\%) and 11 in the other group performed with deep hypothermia and circulatory arrest (10\%). Duration of ECMO was $11 \pm 8$ days. Weaning from ECLS was achieved in 10 patients $(52.6 \%)$, with a survival of $70 \%$. Three patients died after ECLS removal because of septic shock, multi organ failure, and cardiorespiratory failure. Causes of death during ECLS (9 patients) were bronchial/parenchymal bleeding during ECMO in three cases, myocardial infarction in one, cardiac arrest and septic shock in two cases respectively and multi-organ failure in one.

As reported by other authors, our post-PEA ECMO population had a worse preoperative anatomic and hemodynamic profile. Table 1 reports data of ECLS patients compared with non-ECLS patients of an overall cohort of 144 patients with complete data. In the analysis of this group it was evident that most of these patients presented with more severe $\mathrm{PH}$ in terms of PAPs and PVR $(\mathrm{P}=0.046$ and 0.023 , respectively). This was despite being treated with double pulmonary vasodilator therapy (phosphodiesterase- 5 inhibitor and endothelin-1 antagonists) in itself, this could be an indicator of the severity of the pathology and the distal vasculopathy. In Table 1, the hemodynamic profile after the operation is documented. In fact, the group of patients needing ECLS after PEA had a lower reduction of PVR in the first POD. Comparing ECMO survivors and 
Table 2 Clinical, hemodynamics and surgical data of patients with postoperative ECLS. Comparison between survivors and not survivors

\begin{tabular}{|c|c|c|c|}
\hline Variable & ECLS-SV (n=7), N (\%) & ECLS-non SV (n=12), N (\%) & $\mathrm{P}$ \\
\hline BSA & $1.65 \pm 0.16$ & $1.76 \pm 0.25$ & 0.328 \\
\hline Male & $1(14.3)$ & 7 (58.3) & 0.061 \\
\hline NYHA III-IV & $6(85.7)$ & 12 [100] & 0.124 \\
\hline Preoperative vasodilators & $4(57.1)$ & $8(66.7 \%)$ & 0.307 \\
\hline Distal anatomy lesion & $1(14.3)$ & $3(25.0)$ & 0.581 \\
\hline Redo & $3(42.9)$ & $2(16.7)$ & 0.211 \\
\hline Emergency & $0(0)$ & $2(16.7)$ & 0.253 \\
\hline PAPm $(\mathrm{mmHg})$ & $57.29 \pm 10.45$ & $46.75 \pm 13.33$ & 0.092 \\
\hline PVR (WU) & $10.72 \pm 3.04$ & $11.06 \pm 3.91$ & 0.851 \\
\hline $\mathrm{Cl}\left(\mathrm{L} / \mathrm{min} / \mathrm{m}^{2}\right)$ & $2.46 \pm 0.37$ & $2.39 \pm 0.52$ & 0.761 \\
\hline $\mathrm{CO}$ (L/min) & $4.17 \pm 0.60$ & $3.94 \pm 0.99$ & 0.617 \\
\hline CBP time (min) & $322.57 \pm 73.34$ & $351.67 \pm 66.83$ & 0.389 \\
\hline Cross clamp time (min) & $174.00 \pm 43.19$ & $194.17 \pm 42.96$ & 0.338 \\
\hline Circulatory arrest time (min) & $21.29 \pm 26.68$ & $20.92 \pm 23.83$ & 0.975 \\
\hline PVR POD 1 (WU) & $5.15 \pm 2.95$ & $7.39 \pm 6.61$ & 0.414 \\
\hline
\end{tabular}

BSA, body surface area; PAP, systolic pulmonary artery pressure; PAPd, diastolic pulmonary artery pressure; PAPm, medium pulmonary artery pressure; PVR, pulmonary vascular resistance; CI, cardiac index; CO, cardiac output; CBP, cardio-pulmonary bypass; ECLS, extracorporeal life support; SV, survivors; POD, postoperative day; WU, wood unit.

non-survivors, there were not many significant differences (Table 2). Surprisingly, the PAPs $(\mathrm{P}=0.024)$ were lower in the non-survivor group. Hypothetically, this could be a hallmark of worse right ventricular function, with an inability to generate good pulmonary flow and pulsatility.

\section{Discussion}

There is expert agreement that PEA is the treatment of choice in patients with CTEPH $(16,18,19)$. There is also agreement that the diagnostic and therapeutic work- up requires a multidisciplinary approach since it is a rare pathology. Patients present at varying levels of disease progression and recognizing indications for surgical intervention can be difficult. There is a wide spectrum of clinical, hemodynamic, right ventricular function and anatomic features (20). Sometimes, symptoms may appear when the disease is in a very advanced stage, with very high levels of $\mathrm{PH}$ and severe right ventricular dysfunction. In these cases, the risk of complications increases even without any other associated risk factors. To identify patients at risk of postoperative complication is crucial but this is 
not always possible. Recently, we have reported a new radiological score to identify those patients with higher risk of less favorable response to surgery and of course of ELCS (21). This score works by correlating patient CT scan and hemodynamic data. Complications after PEA include persistent $\mathrm{PH}$ with right ventricular and respiratory failure due to reperfusion edema and in a few cases, due to pulmonary hemorrhage. Parenchymal bleeding normally occurs immediately after the operation, so diagnosis is made in the operating theater. The postoperative period is crucial and management often difficult; in some cases, $\mathrm{PH}$ may progressively increase hours later in the ICU due to progressive reperfusion edema. More infrequently, patients may require ECMO pre-operatively due to acute respiratory failure. When conventional medical and respiratory support are not sufficient to stabilize patients, ECLS is a good option to interrupt a vicious circle of increasing PVR, hypoxia, right ventricular failure, low cardiac output and all the obvious adverse conditions.

We agree with other reports (8) that patients needing ECLS can be divided into different groups: (I) those with proximal occlusion and very good surgical results that suffer massive parenchymal edema due to a large reperfused territory. We observed that this occurred more frequently in patients with proximal anatomy and very high level of $\mathrm{PH}$, but our numbers are too small to reach conclusions. (II) Another group of patients are those with a bad preoperative hemodynamic profile: right ventricular failure and distal occlusion, who have minimal decrease of $\mathrm{PH}$ after satisfactory PEA. In these cases, small and peripheral disease is the main cause of failure. (III) Another dramatic scenario is parenchymal bleeding secondary to technical problems and fragility of the denuded vessels, and the presence of areas of infarcted lung parenchyma which may contribute to hemorrhage after reperfusion (11).

ECLS is a well-established therapy in many cardiorespiratory and hemodynamic conditions, thanks to a number of technical and material improvements that allow better coagulation management, less inflammatory response and better gas exchange (22).

ECLS can be setup as: (I) veno-venous (VV); and (II) venoarterial (VA-ECMO). VV-ECMO is a good option in patients with only respiratory failure but preserved right ventricular function. As the group from San Diego reported (4), this approach has many advantages since peripheral cannulation, minimal incision or even percutaneous insertion reduces invasiveness of the procedure. Also, the development of a dual lumen cannula (Avalon Elite, Avalon
Laboratories, Rancho Dominguez, CA, USA) offers the advantage of single site cannulation (23).

VA-ECMO is needed to manage right ventricular dysfunction coupled with hypoxemia. In the setting of $\mathrm{PH}$, the right ventricle initially develops hypertrophy but with the progression and increase of $\mathrm{PH}$, the $\mathrm{RV}$ dilates, loses contractility and systolic function and may also impair left ventricular function (24). With VA-ECMO, the RV is underloaded to facilitate recovery, and pulmonary flow is regulated to reduce RV afterload, avoiding reperfusion damage while maintaining pulsatility and ensuring adequate oxygenation and $\mathrm{CO}_{2}$ levels. Furthermore, the increased afterload of the left ventricle due to arterial inflow of the ECLS, may improve the filling and diastolic performance of the left ventricle, as well as helping to prevent right ventricular ballooning (leftward septal shift).

VA-ECMO cannulation can be peripheral or central. Typically, a peripheral approach has meant femorofemoral (FF) cannulation, however, other cannulation sites such as the subclavian artery can also be used. However, the $\mathrm{FF}$ approach is a straightforward procedure and is easily done under stable conditions while still on CPB. The chest can be definitively closed, reducing the risk of infection and helping cardiac recovery under standard conditions. Moreover, cannulas can be removed in the ICU. Of course, troubles with lower limb ischemia or other vascular complications may occur and should be considered, periodically checked and eventually treated with distal perfusion. Another drawback of the FF-VA-ECMO is the risk of "Harlequin syndrome" development. In these cases, a switch to VV-ECMO or change of cannulation site should be considered if the patient cannot be weaned from ECMO. Other authors prefer central cannulation when ECMO is needed already in the operating room, since the chest is already open and they observed more complications with the peripheral approach.

In cases of pulmonary or bronchial bleeding, VA-ECMO is the first line management option. In these patients, changing from a CPB circuit to a heparin-coated VAECMO circuit, permits full heparin reversal with protamine to arrest bleeding sources. Additionally in these patients, mechanical ventilation has an important role and high PEEP is advisable. Guth et al. reported a series of 8 patients with endobronchial bleeding (2\%) supported with central VA-ECMO using CPB cannulas. Results are outstanding, with 7 patients discharged from hospital.

All previous authors as well ourselves confirm that bleeding, infection and thromboembolic complications are 
the most common complications of ECLS, increasing in frequency with increased duration of therapy. We agree that the patient must be weaned from ECMO as soon as possible. Still, it is very important to recognize early the patient who might need ECLS before hemodynamic and respiratory conditions deteriorate to the point of irreversibility (even with the institution of ECMO). We also agree with other authors that patients with worse hemodynamic profiles and less accessible anatomy, have more risk of requiring ECLS after PEA (6). In those patients, newer technologies like balloon pulmonary angioplasty (PBA) in combination with medical management can be the therapeutic option with the best risk-benefit relationship (25).

\section{Acknowledgements}

None.

\section{Footnote}

Conflicts of Interest: The authors have no conflicts of interest to declare.

\section{References}

1. Martín-Suárez S, González Vargas T, Pacini D, et al. Chronic thromboembolic pulmonary hypertension (CTEPH): Characterization, pulmonary endarterectomy (PEA) and new therapeutic options. Cirugía Cardiovascular 2018;25:93-101.

2. Delcroix M, Kerr K, Fedullo P. Chronic thromboembolic pulmonary hyper- tension: Epidemiology and risk factors. Ann Am Thorac Soc 2016;13 Suppl 3:S201-6.

3. Boulate D, Mercier O, Mussot S, et al. Extracorporeal life support after pulmonary endarterectomy as a Bridge to Recovery or transplantation: lessons from 31 consecutive patients. Ann Thorac Surg 2016;102:260-8.

4. Thistlethwaite PA, Madani MM, Kemp AD, et al. Venovenous Extracorpoeal Life SUpport after pulmonary Endarterectomy: indication, techniques and outcomes. Ann Thorac Surg 2006;82:2139-45.

5. Galiè N, Kim NH. Pulmonary microvascular disease in chronic thromboembolia pulmonary hypertension. Proc Am Thorac Soc 2006;3:571-6.

6. Morsolini M, Azzaretti A, Orlando G, et al. Airway bleeding during pulmonary endarterectomy: the "bubbles" technique. J Thorac Cardiovasc Surg 2013;145:1409-10.

7. Berman M, Tsui S, Vuylsteke A, et al. Successful
Extracorporeal Membrane Oxygenation support after pulmonary Thromboendarterectomy. Ann Thorac Surg 2008;86:1261-7.

8. Ogino H, Ando M, Matsuda H, et al. Japanese Single center experience of surgery for chronic thromboembolic pulmonary hypertension. Ann Thorac Surg 2006;82:630-6.

9. Nierlich P, Ristl R. Perioperative extracorporeal membrane oxygenation bridging in patients undergoing pulmonary endarterectomy. Interact Cardiovasc Thorac Surg 2016;22:181-7.

10. Manecke GR, Kotzur A, Atkins G, et al. Massive pulmonary hemorrhage after pulmonary thromboendarterectomy. Anesth Analg 2004;99:672-5.

11. Guth S, Wiedenroth CB, Wellenschlages M, et al. Shortterm venoarterial extracorporeal membrane oxygenation for massive endobronchial hemorrhage after pulmonary endarterectomy. J Thorac Cardiovasc Surg 2018;155:643-9.

12. Chacón-Alves S, Pérez-Vela JL, Grau-Carmona T, et al. Veno-arterial ECMO for rescue of severe airway hemorrhage with rigid bronchoscopy after pulmonary artery thromboendarterectomy. Int J Artif Organs 2016;39:242-4.

13. Mikus PM, Mikus E, Martìn-Suàrez S, et al. Pulmonary endarterectomy: An alternative to circulatory arrest and deep hypothermia: mid-term results. Eur J Cardiothorac Surg 2008;34:159-63.

14. Galiè N, Humbert M, Vachiery JL, et al. 2015 ESC/ERS guidelines for the diagnosis and treatment of pulmonary hypertension The Joint Task Force for the Diagnosis and Treatment of Pulmonary Hypertension of the European Society of Cardiology (ESC) and the European Respiratory Society (ERS). Eur Respir J 2015;46:903-75.

15. von Segesser LK, Kwang K, Tozzi P, et al. A simple way to decompress the left ventricle during venoartiral bypass. Thorac Cardiovasc Surg 2008;56:337-41.

16. Frenckner B, Broman M, Broomé M. Position of draining venous cannula in extracorporeal membrane oxygenation for respiratory and respiratory/circulatory support in adult patients. Crit Care 2018;22:163.

17. Kang G, Ha R, Banerjee D. Pulmonary artery pulsatility index predicts right ventricular failure after left ventricular assist device implantation. J Heart Lung Transplant 2016;35:67-73.

18. Jenkins D, Madani M, Fadel E, et al. Pulmonary endarterectomy in the management of chronic thromboembolic pulmonary hypertension. Eur Respir Rev 2017;26(143).

19. Madani MM. Surgical treatment of chronic 
thromboembolic pulmonary hypertension: Pulmonary thrombo endarterectomy. Methodist Debakey Cardiovasc J 2016;12:213-8.

20. de Perrot M, Mayer E. Chronic thromboembolic pulmonaryhypertension: do we need a new definition? Eur Respir J 2014;44:1401-3.

21. Leone MB, Giannotta M, Palazzini M, et al. A new CTscore as index of hemodynamics changes in patients with chronic thromboembolic pulmonary hypertension. Radiol Med 2017;122:495-504.

22. Donahoe L, Granton J, Mc Rae K, et al. Role of extracorporeal life support after pulmonary

Cite this article as: Martin-Suarez S, Gliozzi G, Fiorentino M, Loforte A, Ghigi V, Di Camillo M, Galiè N, Pacini D. Role and management of extracorporeal life support after surgery of chronic thromboembolic pulmonary hypertension. Ann Cardiothorac Surg 2019;8(1):84-92. doi: 10.21037/ acs.2019.01.02 endarterectomy: a single-center experience. Interact Cardiovasc Thorac Surg 2016;23:74-8.

23. MacLaren G, Combes A, Bartlett RH. Contemporary extracorporeal membrane oxygenation for adult respiratory failure: life support in the new era. Intensive Care Med 2012;38:210-20.

24. Machuca TN, de Perrot M. Mechanical support for the failing right ventricle in patients with precapillary pulmonary hypertension. Circulation 2015;132:526-36.

25. Madani M, Ogo T, Simonneau G. The changing landscape of chronic thromboembolic pulmonary hypertension management. Eur Respir Rev 2017;26(146). 